\title{
Chronic pain: from wait lists to rehabilitation, a clinical report of the Manitoba perspective
}

\author{
Ryan J. Amadeo, MD • Eric Sutherland, MD
}

Received: 8 December 2009/Accepted: 7 January 2010/Published online: 20 January 2010

(C) Canadian Anesthesiologists' Society 2010

\section{To the Editor,}

Chronic pain is recognized widely for its personal and societal burden, ${ }^{1}$ and it remains an elusive target for treatment success. While much has been learned about the pathophysiology, psychology, and medical therapeutics of pain, patients continue to languish awaiting entry into multidisciplinary pain clinics throughout Canada. ${ }^{2,3}$ Recent international publications have gone so far as to declare access to pain management as a fundamental right to be adhered to in the developed and developing world. ${ }^{4}$

Based on data collected from June 2005 to August 2006, Peng et al. surveyed access to Canadian Multidisciplinary Pain Clinics (MPC). Funding source (public or private) and province-of-service provision were independent predictors for MPC access times. With a solely publicly funded MPC, the wait time in Manitoba was the longest of the ten provinces. The Canadian Pain Society Wait Times Task Force Statement recommends wait times of no longer than six months for initial assessment at a MPC.

Initiatives developed within the Pain Management Centre at the University of Manitoba allowed our MPC to reduce wait times to three months. The previous wait time for new referrals to enter our MPC averaged 33-months. Patients entered the MPC and were assessed as a "new consultation" by an anesthesiologist. The anesthesiologist would manage the patient's treatment and consult with other practitioners. These practices ran in parallel, and anesthesiologists would cover for each other when their colleagues were not present in the clinic.

After "follow-up consultation" for patients already within the clinic system, new patients were scheduled into

R. J. Amadeo, MD $(\varangle)$. E. Sutherland, MD

University of Manitoba, Winnipeg, Manitoba, Canada

e-mail: ramadeo@shaw.ca the clinic as space allowed. A priority system allocated space to urgent pain categories first, including cancer pain, complex regional pain syndrome, and post-herpetic neuralgia. There was one physician clinic per day and one physician procedure clinic (fluoroscopic-guided procedures) per day. Nursing resources contributed to managing patients scheduled in clinics, with one nurse per day assigned to respond to telephone calls from patients (approximately 50-80 calls per day).

Prior to restructuring, our clinic had approximately 5,000 active patients under care at any given time. We have increased our new patient visits from 550 to 1,050 per year (91\% increase), and our follow-up visits have increased from 3,168 to 5,750 per year ( $82 \%$ increase). The two cornerstones of our practice restructuring are complete group practice, with each physician sharing responsibility for every patient, and a partnership with family physicians permitting patients to return to primary care when they have completed the patient navigation pathway.

Patients enter the MPC and are assessed as a "new consultation" by an anesthesiologist who initiates a management course that includes medication titration, interventional management, and additional therapy with physical medicine, physiotherapy, and psychology. In order to standardize and facilitate timely methodical access to treatments, our clinic physicians have developed procedurebased algorithms that are tailored to individual patient needs.

The current clinic infrastructure has added a second satellite site with a Pain Management Specialist present each day. At this second site we have added additional psychology, physiotherapy, and nursing resources. We are also adding a primary care physician to our model to assist in de-intensifying patients' care as a step toward discharge back to their family physician. Our current and previous staff resources are reflected in the Table 1. 
Table 1 Pain clinic resources before and after restructuring

\begin{tabular}{|c|c|c|c|c|c|c|}
\hline \multirow[t]{2}{*}{ Discipline } & \multicolumn{3}{|l|}{ Full-time equivalent } & \multicolumn{3}{|l|}{ Number of practitioners } \\
\hline & Before re-structuring & After re-structuring & $\%$ Change & Before re-structuring & After re-structuring & $\%$ Change \\
\hline Anesthesiology & 2 & 3 & 50.0 & 6 & 6 & 0.0 \\
\hline Physical med and rehab & 0.6 & 0.6 & 0.0 & 3 & 3 & 0.0 \\
\hline Psychology & 0.6 & 1.6 & 166.7 & 2 & 3 & 50.0 \\
\hline Physiotherapy & 0.4 & 0.9 & 125.0 & 1 & 2 & 100.0 \\
\hline Nursing & 3 & 5 & 66.7 & 4 & 6 & 50.0 \\
\hline Radiation technology & 1 & 1 & 0.0 & 2 & 2 & 0.0 \\
\hline Administrative staff & 3 & 4.5 & 50.0 & 3 & 5 & 66.7 \\
\hline Total staffing & 10.6 & 16.6 & 56.6 & 21 & 27 & 28.6 \\
\hline
\end{tabular}

At our primary clinic site, we have restructured the flow of patients significantly to allow for timely methodical access to resources. Each clinic schedules three to four new patients prior to adding "follow-up patient visits" - the reverse of our previous system. We currently have, on average, a three- to five-month wait for new referrals to enter our MPC. Our daily clinics are structured as follows: In the Titration Clinic, patients entering the clinic receive a rational approach to medication trial. Medications are trialed one at a time and titrated to an endpoint of analgesic success, failure, or side effects. The patient has additional appointments scheduled approximately two weeks apart. In the Fluoroscopy and Procedure Clinic, one physician performs procedures each day that are tailored to individual patient needs, including procedures in the fluoroscopy suite and more invasive pain procedures in the general operating room.

Our current goal is to care for patients in our Pain Clinic for three to six months, then discharge them back to primary care so as to allow additional patients to enter our clinic. We partner with family physicians to provide ongoing patient support.

In conclusion, chronic pain is well recognized for its societal burden and prevalence. Current evidence suggests that targets set for access to care within a multidisciplinary pain clinic are not being met within the public health care system in Canada. With a modest increase in resources, we have shown that a proportionally greater increase in efficiency can be accomplished with innovations to practice patterns. We have achieved this goal in a health care system without private clinics - a model where such improvements were previously deemed impossible.

Acknowledgements We acknowledge and thank Ms. Linda Girling and Dr. W.A.C. Mutch for their advice and assistance in preparing this letter for publication.

Disclosure The authors have no disclosures.

Competing interests None declared.

\section{References}

1. Thomsen AB, Sorensen J, Sjogren P, Eriksen J. Chronic nonmalignant pain patients and health economic consequences. Eur J Pain 2002; 6: 341-52.

2. Peng $P$, Choiniere $M$, Dion $D$, et al. Challenges in accessing multidisciplinary pain treatment facilities in Canada. Can J Anesth 2007; 54: 977-84.

3. Morley-Forster PK. Tomorrow and tomorrow and tomorrow: wait times for multidisciplinary pain clinics in Canada. Can J Anesth 2007; 54: 963-8.

4. Brennan F, Carr DB, Cousins M. Pain management: a fundamental human right. Anesth Analg 2007; 105: 205-21. 